\title{
A Review of Hydrogels, Their Properties and Applications in Medicine
}

\author{
Hossein Chamkouri ${ }^{1,2 *}$ and Mahyodin Chamkouri ${ }^{2}$ \\ ${ }^{1}$ Faculty of Chemical engineering, University of Tabriz, Iran \\ ${ }^{2}$ Danesh Paper Company, Iran
}

*Corresponding author: Hossein Chamkouri, Faculty of Chemical engineering, University of Tabriz, Danesh Paper Company, Bushehr, Iran.

To Cite This Article: Hossein Chamkouri, Mahyodin Chamkouri. A Review of Hydrogels, Their Properties and Applications in Medicine. Am J Biomed Sci \& Res. 2021 - 11(6). AJBSR.MS.ID.001682. DOI: 10.34297/AJBSR.2021.11.001682.

Received: 畊 January 25, 2021; Published: 眥 February 03, 2021

\begin{abstract}
A hydrogel is a water-insoluble three-dimensional polymer network that has the ability to absorb body fluids in a biological environment. Such a polymer network is formed through chemical crosslinking mechanisms such as optical polymerization, enzymatic reactions and physical crosslinking such as temperature and $\mathrm{pH}$ dependent processes and ionic crosslinking. Physical hydrogels are formed through weak secondary force and chemical hydrogels are formed by covalent forces. Various polymers of natural and synthetic origin are used to make hydrogels. Swelling, mechanical properties and biological properties are among the most important properties of hydrogels, each of which can affect the structure and morphology of the hydrogel. Due to its structure similar to the extracellular matrix (ECM) and its ability to absorb water, hydrogen is used in various medical applications such as tissue engineering, contact lenses, wound dressings, and release of therapeutic agents. Hydrogels, types of hydrogels, their properties and applications in medicine are discussed.
\end{abstract}

\section{Introduction}

Much scientific research has been done in the field of biomaterials that play a role in human health [1]. In this article, we review hydrogels, which is one of the main topics in biomaterials research. Hydrogels are water-insoluble three-dimensional polymer networks that have the ability to absorb large amounts of water or biological fluid in the body due to the presence of hydrophilic groups in their structure [2-5]. Among the hydrophilic functional groups in the main polymer chain of hydrogels, we can mention the hydroxyl groups ( $\left.\mathrm{OH}_{-}\right)$, carboxyl ( $\left.\mathrm{COOH}-\right)$, amine $(\mathrm{NH} 2)$, and sulfate $\left(\mathrm{SO}^{-} \mathrm{H}_{-}\right.$ ) [6]. Polymeric hydrogels can be formed by physical crosslinking, chemical crosslinking, or a combination of both types [7].

The chemical structure of the hydrogel, its morphology and equilibrium swelling affect properties such as mechanical strength and intracellular and extracellular transport [1]. Hydrogels due to water absorption, soft structure, biocompatibility, low protein adsorption due to low surface tension and similarity to ECM structure, special attraction for various medical applications including tissue engineering, release of therapeutic agents (proteins, drugs, genes) Contact lenses and wound coverings [6-10]. Hydrogels can be made from several perspectives, including the main source of the hydrogel (natural or synthetic polymer (hydrogel structure), homopolymer network, copolymer network and permeable network. Physical and chemical cross-links (anionic and cationic (and biodegradable) hydrogen gel loads (and biodegradable) Biodegradable, biostable (divided by $[1,4]$. Recently, researchers have paid more attention to Hydrogels that respond to biological conditions [11]. Using this type of hydrogel, also called injectable hydrogen, the patient gets rid of the complications of surgery for implantation, such as pain and inflammation. Hydrogels are prepared at the desired location in the body according to biological conditions [6].

In such systems, the polymer solution is injected into the body in liquid form and converted into solid hydrogel using physical and chemical crosslinking agents [6,7]. Stimulating factors for the formation of hydrogels include changes in temperature, $\mathrm{pH}$, the pres- 
ence of ions and ultraviolet radiation $[12,13]$. The crosslinking process affects factors such as molecular mass, mechanical strength, and heat and solvent resistance. Both physical and chemical crosslinking create a three-dimensional structure of the hydrogel that allows therapeutic agents (drugs and biomolecules) to be trapped and released from the structure [1]. The properties of hydrogels and their application in various medical fields are discussed for instance Hydrogels vary in dimension, function and architecture would be effective in drug delivery (Figure 1).

\section{$\begin{array}{lll}\text { Hydrogel } & \text { Meshes } & \text { Polymer drug interactions }\end{array}$}

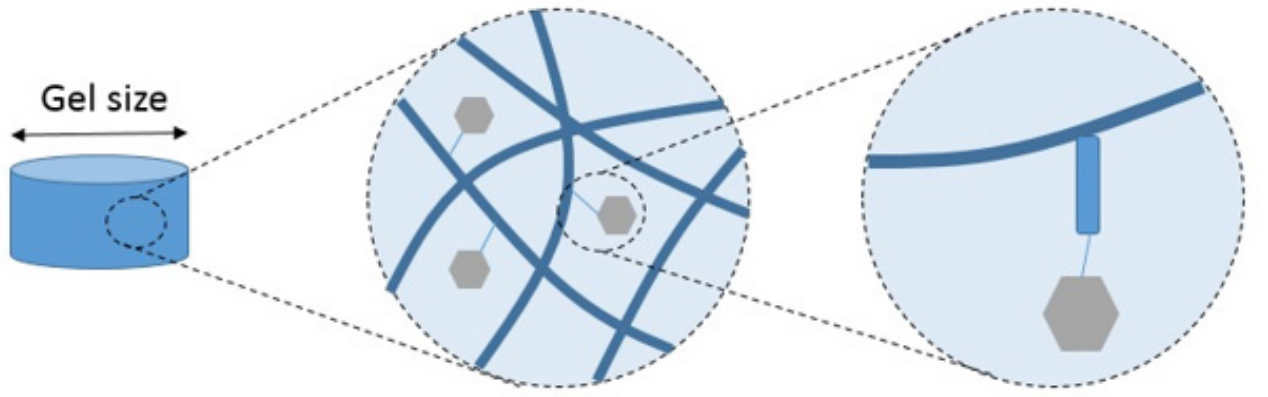

\section{$\mathrm{Cm}$}

$\mu \mathrm{m}$

$\mathrm{nm}$

Figure 1: Multiscale properties of hydrogels.

\section{Mechanism of Hydrogel Formation}

Polymers are carbohydrate materials that have been widely used to make physical and chemical hydrogels due to their availability, presence of modifiable functional groups, biocompatibility and other properties [6]. Hydrogels can be designed for a specific application by selecting the type of monomer or polymer and the type of hydrogel formation reactions. Hydrogels are formed by two methods of chemical crosslinking and physical crosslinking [14].

\section{Chemical Crosslinking}

Chemical cross linkable hydrogels are a type of hydrogel that can be converted from a liquid to a solid by covalent bonding. This method is also used in in situ hydrogel systems. In this method, various reactions such as optical polymerization, enzymatic reactions and click reactions are used to form hydrogels. In this section, the mentioned methods for making these hydrogels will be discussed [15]. Hydrogels formed by chemical crosslinking have been considered because of their good mechanical strength [16].

\section{Optical Polymerization}

Optical polymerization is one of the methods of chemical crosslinking in the formation of hydrogen that has advantages such as low energy and no need for solvent to perform the reaction. This method uses hydrophilic polymers that have light-sensitive molecules. When the polymer solution is exposed by UV or visible light, the optical decomposition initiator and free radicals are formed and the polymerization process takes place. Polymers that crosslink in this way usually have groups of acrylates and methacrylates, which are polymerized by light. The rate of gelling in this method can be controlled and this hydrogen can be used to release therapeutic agents such as proteins and drugs [15]. This method is one of the most effective ways in which a monomer is converted to a polymer through an optical initiator [17]. Optical polymerization strengthens the hydrogel and allows the cells to fade during gel formation, which is used for clinical applications. This method also creates a porous lattice structure for the hydrogel [18]. Optical polymerization is easily performed at physiological temperature and $\mathrm{pH}$ inside or outside the body. For hydrogels used in the medical field, a non-toxic optical primer with appropriate light wavelength radiation from a light source should be used for example a mechanism of gelling by optical crosslinking through the failure of vinyl groups in contact with UV radiation [19].

\section{Enzymatic reactions}

Enzymatic reactions take place in the presence of enzymes in the biological environment. Therefore, this method has attracted much attention for cellular applications. Optimal cross-linking conditions such as natural $\mathrm{pH}$, biological environment and optimum temperature are required to crosslink through enzymatic reactions. An important advantage of this method for the formation of hydrogels is the special enzyme substrate that has the ability to prevent 
the entry of toxic substances caused by side reactions. Horseradish peroxidase (HPR) Modified glutamines and tyrosinases are among the substances used as enzymatic catalysts in the preparation of hydrogel systems used in tissue engineering. HPR is one of the enzymes that due to its high mechanical stability and easy purification, is used to form hydrogels in this method and therefore it is used in various medical applications such as drug release, reconstruction and repair and tissue engineering. The HPR-H2O2 enzyme water system is widely used in the formation of natural hydrogels such as chitosan, hyaluronic acid, dextran and gelatin to form hydrogels. In a research work by Kurosawa et al. It was prepared by enzymatic reactions and used to release therapeutic proteins. In this study, hyaluronic acid was functionalized using tyramine and labeled with the fluorescent label amino fluorescein. The subcutaneous gelling time was reduced, so the use of enzymatic reactions to establish cross-linking due to its selectivity as well as cell compatibility is a suitable method for hydrogel formation.

\section{Click reaction}

Click chemistry, as defined by Sharples et al., Is defined as certain types of reactions in the presence of a catalyst that have high speed and efficiency, excellent biological properties, and favorable reaction conditions $[1,20]$. Click chemistry plays a significant role in the synthesis and activation of polymers and is used as an effective and flexible method for functionalizing molecules. Due to the benefits of click chemistry, this method is used to make hydrogels, nanogels and microgels.

It has also been used as a substrate for tissue engineering and drug delivery. Click chemistry is known as an emerging substrate for polysaccharide-based hydraulic chemical crosslinking. Click reactions generally include a wide variety of reactions, including: copper (I) reactions (catalyzed by alkyne azide sequestered, catalytic reactions of free pair of alkyne azides, silicosterone reaction with disaccharide (DA), among which the intergroup reaction Alkynes and azides are the most obvious examples of click chemistry due to their advantages such as high efficiency in physiological conditions of the body and high selectivity [1]. In the absence of side-products that are thermally reversible and allow the degree of reaction to be controlled, the reaction takes place in the absence of a catalyst or primer and protects the biocompatibility of the material [20]. Starch-based hydrogels were synthesized by click reaction between thiol and allyl groups of starch for biomedical and tissue engineering applications. The resulting hydrogel showed good swelling behavior and biodegradability [21].

\section{Physical Crosslinking}

Hydrogels formed by physical bonding can be obtained by modifying intramolecular forces such as hydrogen bonding, hydrophobic interaction, and electrostatic ionic force. In order to prevent the possible increase in the toxicity of the crosslinker in the chemical method, this method makes it possible to prepare hydrogel with simple and safe processes. Physical cross-linking methods include ionic methods, temperature-dependent methods and pH-dependent methods for cross-linking [20]

Ionic crosslinking: Among the physical methods of crosslinking, the ion crosslinking reaction is released without the formation of a covalent bond between the polymer chains, in which the ion crosslinking agent is used to form the gel $[6,13]$. This method is used to achieve high toughness for hydrogels [22]. Alginate is a natural polysaccharide and its polymer solution has the ability to form gels against divalent cations such as $\mathrm{Ca} 2+$. These ions lead to the bonding of gluronic acid groups in the alginate chain and the formation of ionic bonds within the polymer chain. Hydrogen alginate is commonly used as an ECM. Recently, researchers have focused on optimizing gelling time, their mechanical properties, as well as improving biological interactions. By controlling parameters such as molecular weight, concentration of alginate and calcium and the composition of alginate in the made hydrogel, they can be prepared for use in injectable biomaterials and cultured in vitro $[13,14]$. Other natural polymers that have the ability to crosslink ionically include chitosan, which has been used in applications such as drug release [7].

Temperature dependent methods: Temperature is an important parameter in the formation of hydrogen by physical crosslinking. Temperature-sensitive hydrogels become liquid at low temperatures and gels at body temperature [23]. Water-soluble polymers have the property of gelling under temperature changes, and these hydrogels have been used for tissue engineering applications. These hydrogels do not need any chemical stimulants to form. Their gel point can be adjusted to a temperature close to normal body temperature, so they can be injected into a liquid and solidified inside the body. Both natural and synthetic polymers are used in this method. Natural polymers include cellulose, chitosan and gelatin derivatives, and synthetic polymers include poly-isopropyl acrylamide (PNIPAAM) and its copolymers as well as pluxamer. Recent research on the use of PNIPAAM copolymers in tissue engineering applications has been concentrated for the release of chondrocytes and growth factors, and ploxamers have also been tested for lung tissue engineering. Physical gelling of ploxamer with a chemical crosslinking agent has been used to improve mechanical properties to trap cells. Also, temperature-sensitive and injectable hydrogel of hyaluronic acid has been considered by researchers due to its high biocompatibility and high sensitivity to body temperature [24].

pH-dependent methods: It should be utilized as a hydrogel stimulant because every position of the human body is related to a specific $\mathrm{pH}$ [15]. Among stimulus-responsive hydrogels, pH-sen- 
sitive hydrogels have been widely used in studies and research pH-sensitive hydrogels have the potential to swell and contract dynamically due to the average $\mathrm{pH}$ of their surroundings. A common feature of $\mathrm{pH}$-sensitive hydrogels is the presence of side acidic groups that can be ionized at a specific $\mathrm{pH}$. $\mathrm{pH}$-sensitive hydrogels have been widely used in medical applications such as controlled drug delivery systems and heart valves due to their ability to stimulate and respond to environmental changes. In some applications, especially drug release, hydrogels are exposed to different temperatures in the body. To understand the formation of $\mathrm{pH}$-sensitive hydrogels, temperature changes must be made [15]. In a research study by Hu et al. Carboxymethyl chitosan was used along with an unstable acid crosslinking agent under optimal conditions to make pH-sensitive hydrogels. Carboxymethyl chitosan is a non-toxic, biodegradable and biodegradable substance. It is compatible and has been widely utilized in medicine and medicine [25].

\section{Types of Hydrogels}

Depending on the type of polymer, hydrogels are divided into two categories: natural and synthetic hydrogels. Hydrogenated by natural or synthetic polymers are considered as raw materials for medical applications. Natural and synthetic polymers used to make hydrogels must be biocompatible, biodegradable, and in some applications where the hydrogel is in contact with the blood must be blood compatible [26].

\section{Natural Hydrogels}

Natural hydrogels are gels whose polymers are obtained from natural sources. The use of natural polymers to form hydrogels has advantages such as biocompatibility, biodegradability and non-toxicity. The use of natural polymers in the manufacture of hydrogels depends on the purpose of the use of biomaterials. For example, hydrogels used for controlled release of materials must be biocompatible, biodegradable, and informal [27]. Natural polymers include polysaccharides and their proteins, which are widely used as carriers for the release of substances. The results of in-body testing of these polymers showed their biocompatibility, among which, polysaccharides are more suitable due to biocompatibility, enzymatic degradation, high durability and non-toxicity [28]. Natural hydrogels used in clinical applications include alginate, collagen, gelatin, and fibrin. Alginate, for example, has been used to return left ventricular activity to normal after a heart attack. Collagen has also been used as an alternative to vascular bundles. Gelatin can be used for artificial structures and fibrin can be used for tissue engineering as well as adhesives and anticoagulants during surgery [29].

\section{Synthetic Hydrogels}

Synthetic hydrogels are obtained from synthetic polymers such as polyamides or polyethylene glycol (PEG). More recently, synthetic polymers have been substituted for natural polymers in hydrogel construction due to advantages such as long life, gel strength, and greater ability to absorb water. Synthetic polymers used to make hydrogels are used in various medical applications. Synthetic polymers are hydrophobic and in terms of mechanical structure and Chemicals are better than natural polymers. These polymers include polyacrylamide and its derivatives, polyvinyl alcohol and PEG. PEG is one of the most popular polymers used for synthetic hydrogenation in various medical applications such as drug release, tissue engineering, bone prostheses and wound dressings. This polymer is used for various medical applications due to its properties such as biocompatibility, non-stimulation of the immune system and resistance to protein adsorption. PEG alone has the ability to form insoluble network structures. However, adding factor groups to it improves the crosslinking in the structure of the hydrogen network [27].

\section{Properties of Hydrogels}

Hydrogels are network polymers that have the ability to absorb and retain water, and this property causes the hydrogel to swell [30].

\section{Swelling of hydrogel}

Equilibrium swelling of hydrogels is obtained from the following formula:

$$
\text { swelling ratio }=\left(w_{-} t-w_{-} d\right) / w_{-} d
$$

Where $\mathrm{W}_{\mathrm{t}}$ is the weight of the swollen hydrogels and $\mathrm{Wd}$ is the weight of the dried hydrogels in freeze-drying. In standard hydrogel systems, drug release occurs mainly through swelling or contraction of the hydrogel and diffusion of the drug through the polymer network. When the properties of a hydrogel such as swelling behavior, structure, mechanical strength or permeability can change in response to various stimuli, it can be called a stimulus-responsive hydrogel or a biologically sensitive hydrogel. These agents can be used effectively in altering the release of drug agents from hydrogels for the controlled release of drug agents [6]. Figure 2 shows the hydrogen swelling ratio of adipic acid dehydrated / polyglutamic acid and hyaluronic acid. All hydrogen has a high inflation rate and the highest inflation rate can reach $70 \%$. Figure 2 shows that when the solid content was $1 \%$ by weight, the hydrogel was dissolved in buffer phosphate salt solution. Using copolymerization of hyaluronic acid and polyglutamic acid, Xiobin, et al. Prepared a high-swelling injectable hydrogel to control protein release. As the weight percentage of polymer components increased, the percentage of swelling decreased, which was associated with an increase in crosslinking in the structure. Their research also showed that the $\mathrm{NH} 2$ / CHO molar ratio can affect the percentage of hydrogel swelling. As shown in Figure 2 when the NH2 / CHO molar ratio reached $1: 2$, the swelling rate was about twice that of the $2: 1$ hydrogel, and when it was 1: $3 \mathrm{NH} 2$ / $\mathrm{CHO}$, due to the low rate. Cross-linking in 
structure, hydrogel was dissolved in buffer phosphate salt solution (PBS) [24].

The swelling behavior of $\mathrm{pH}$-sensitive polymers has been used for the controlled release of drug agents. The molecular weight of a polymer and the crosslinking between the two points are the most important features of the swelling behavior, the release rate Controls drug and oxygen permeability [14]. Hydrogens are able to swell in biological environments and retain large amounts of water. Cross-linking between structure chains prevents dissolution and retains the dimensions of the network. The hydrogel is highly dependent on external factors such as temperature, $\mathrm{pH}$, ion concentration, etc. which in turn can contribute to the collapse or phase transfer of the hydrogel. Drug release is mainly done by swelling of a hydrogel and drug release can be by Inflation, diffusion, or both can be controlled by adding PEG to the PNIPAAM polymer system to improve the swelling ability of the copolymer [12].

\section{Mechanical Properties}

Injectable chemical hydrogels have better mechanical properties and longer stability than physical hydrogels, but the use of toxic crosslinking agents in chemical hydrogels for preparation can have a negative effect on biocompatibility, while in hydrogels Physical-chemical interactions are established and they avoid such toxic initiators [6]. The mechanical properties of hydrogels are considered as one of the important parameters for engineering design of hydrogels for medical applications. In the field of tissue engineering, the mechanical properties of scaffolds at both macroscopic and microscopic scales play an important role in regulating cell behavior.

Biomechanical signals and interactions between cells and ECM directly affect the shape of cells. For example, ECM stiffness affects adult human skin fibroblasts. Also, cells exposed to stronger substrates organize a higher elastic modulus in the plasma membrane with a better cytoskeleton. In addition, cells cultured on harder substrates have a faster rate of proliferation and migration than softer substrates. The mechanical properties of hydrogel biomaterials are primarily defined using time-independent and time-dependent viscoelastic theories to analyze the structure of the hydrogel and estimate the effective crosslinking ratio (Figure 3) [31]. PNIPAAM hydrogel prepared by free radical polymerization method is very weak and standard mechanical identification methods cannot be used to determine their mechanical properties. Some of the parameters affecting the mechanical properties of hydrogels are the initial monomer concentration, the amount of crosslinking agent, the polymerization temperature, the degree of swelling at the time of measurement and the method of measurement. This makes it very difficult to accurately compare any mechanical parameter [32]. Reza Abdollahi and colleagues used graphene oxide to enhance the mechanical properties of amylose-bonded PAA. They believe that graphene oxide has a suitable strengthening effect for tensile properties due to its wide aspect ratio and distinct mechanical strength.

\section{Biological Properties}

Essential properties of injectable hydrogels for various medical applications include biocompatibility and non-toxicity, sufficient mechanical properties, good viscosity, stability, biodegradability, etc. The hydrogel must have suitable mechanical and biological properties, and these properties must be similar to the tissue that replaces it [6]. In several studies, Zhao et al. Have tried to achieve optimal mechanical and biological properties by combining cross-linking and electrospinning methods. Electrospun gelatin methacrylate fibers were crosslinked using UV light. The results showed that the physical and biological properties of electro spun hydrogel can be changed by changing the exposure time [33]. To mimic real bone tissue, it is important to develop a hydroxyapatite / carbon nanotube / HA CNT composite with excellent mechanical and biological properties for the use of bone substitutes.

The special top of the composite not only helps the circulation of body fluids, but also shows a moist and uneven surface, which is a desirable feature for cell adhesion, proliferation and growth. Therefore, HA / CNT composite with excellent biological and mechanical properties has great potential for use in bone replacement applications and highly active tissue scaffold preparation. Cell expansion in biological material is an important step for the basic biological properties of osteoblasts [34]. The development of an injectable hydrogel, its biocompatibility should be examined because the hydrogel should support the growth and differentiation of cells without causing toxicity or immune reactions in the host. Because most of the components of natural hydrogels are similar to ECM, their biocompatibility it is more than artificial hydrogels, the structure of the hydrogel must be compatible with cells, tissues and body fluids, it must be non-toxic and non-cancerous, and it must not cause chronic physiological or inflammatory reactions after its destruction [6].

\section{Application of Hydrogels in Medicine}

\section{Tissue Engineering}

Hydrogels of highly hydrated polymer networks are similar to ECMs, which have attracted much attention for tissue engineering and regenerative medicine applications. To date, various types of hydrogels derived from natural or synthetic polymers have been used to reconstruct defective osteocortical joints or articular cartilage tissue. Alginate is a natural polysaccharide polymer that is usually obtained from brown seaweed and various bacteria. One of the unique features of alginate is the ability to physically cross-link by divalent cations such as $\mathrm{Ca} 2+$ at room temperature, which makes it very useful in various biotechnical methods such as molding, spraying or 3D printing. 


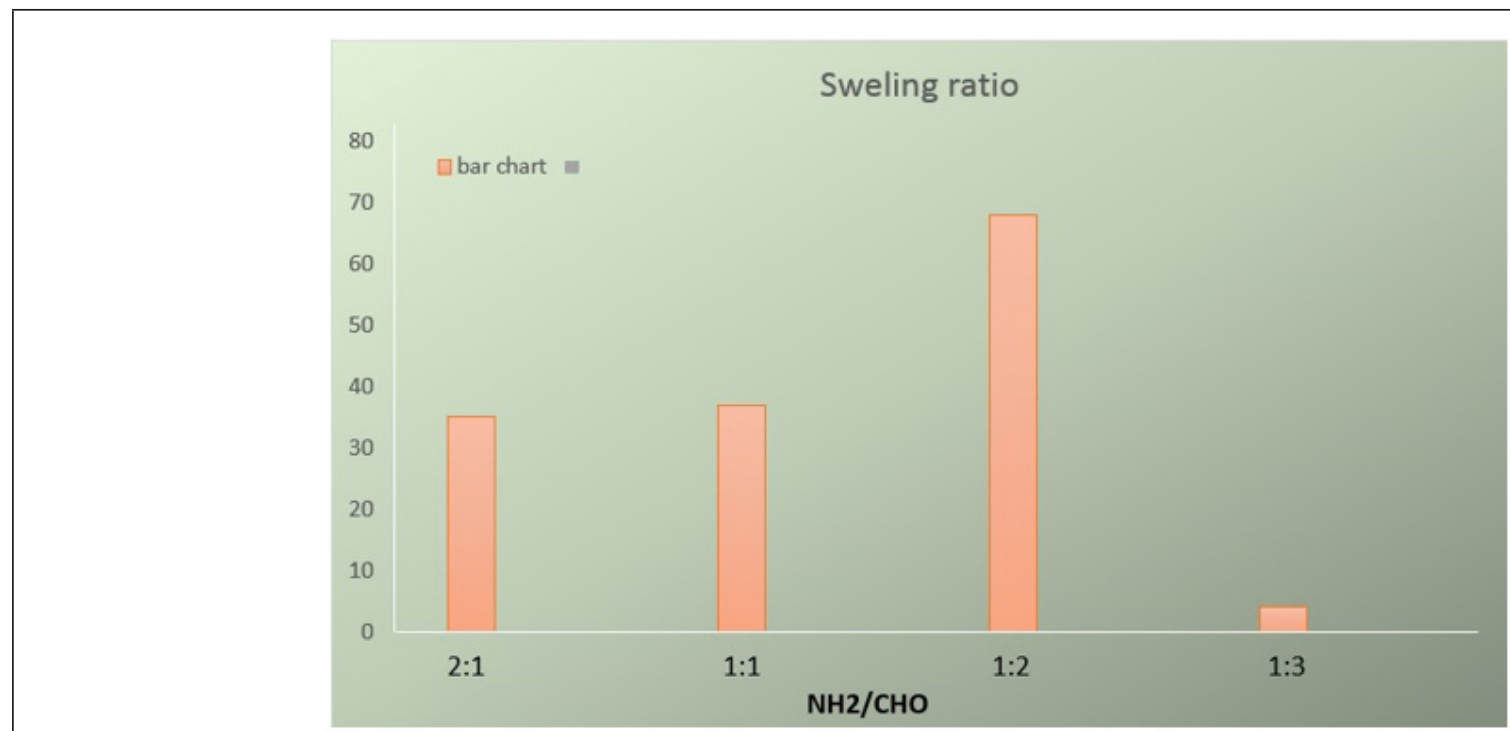

Figure 2: Swelling ratio of dehydrated adipic acid / polyglutamic acid and hyaluronic acid hydrogels [24].
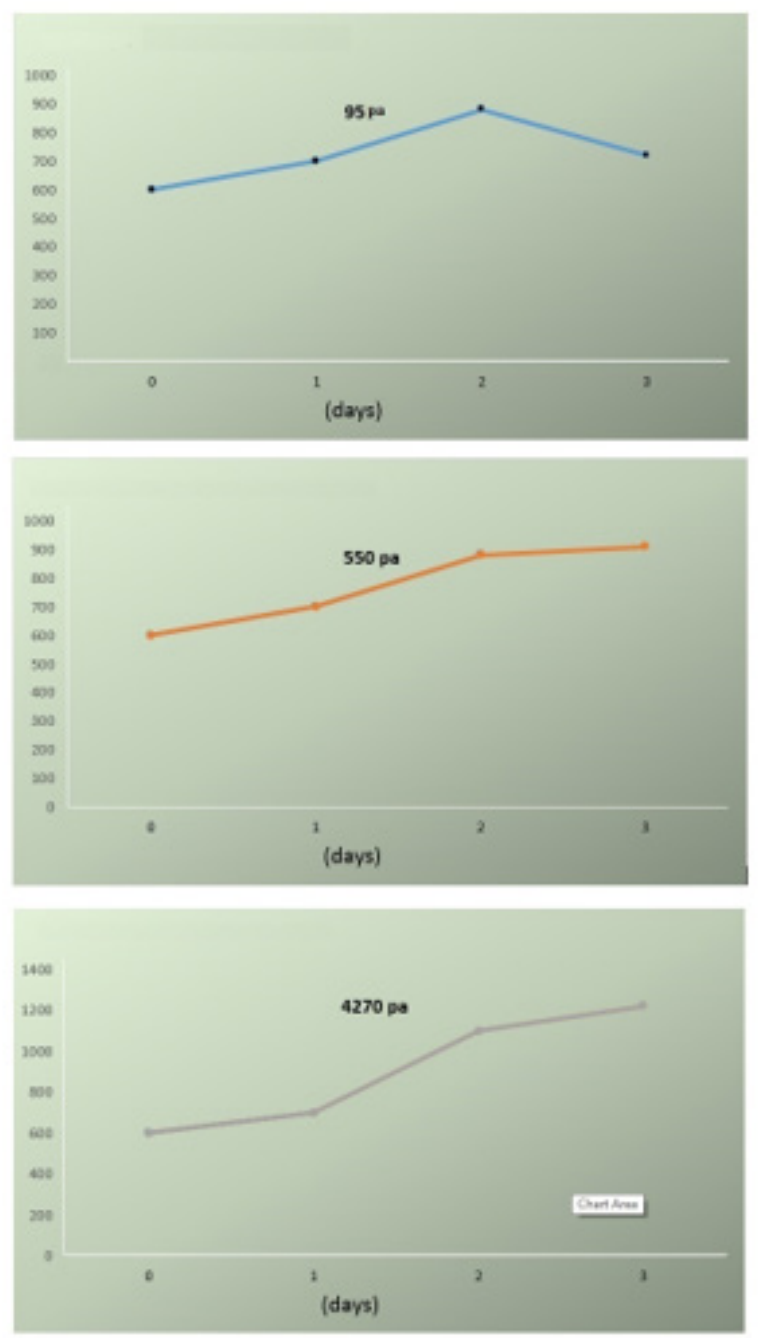

Figure 3: Comparison diagram of hydrogel stiffness with respect to cell proliferation [31]. 


\section{Physical Hydrogels}

The products have good biocompatibility, low toxicity and relatively low cost. Alginate hydrogel is suitable for supporting the growth and proliferation of encapsulated chondrocytes as well as maintaining the chondrogenic phenotype. After inoculation of chondrocytes for 21 to 28 days, type II collagen is formed along with the advanced cartilage gene. Alginate is also used to provide primary bone cells, including chemical stem cells (MSCS), for bone regeneration. Encapsulated MSCS can produce their own collagen ECM that integrates with host tissue.

It is the second most abundant natural biopolymer obtained from renewable sources such as oyster shells, insects, fungi and marine waste. Chitosan has good biocompatibility and biodegradability and is therefore a suitable material for tissue engineering applications. Chitosan produced by enzymatic grafting can support the proliferation of chondrocytes and stem cells.

Maintain chondrogenic phenotype and morphology and increase ECM deposition in vitro [ECM.] 36 has long been recognized as a support material produced by cells as the only scaffold on which cells rest. Neutral materials have better biocompatibility than other materials and due to their ineffective properties, they can be used for many applications, including tissue engineering, replacing the artificial hip joint [36]. Alexander et al. were able to provide temperature-sensitive injection hydrogels for tissue engineering applications by copolymerizing PEG and PNIPAAM. They believe that PNIPAAM-based hydrogels have low compressive modulus and low mechanical properties. Due to the temperature sensitivity of PNIPAAM polymer, this material is a suitable candidate for the development of an injectable biological material for use in the regeneration or replacement of soft tissue [12].

Hydrogels made from natural materials have often been used as tissue engineering scaffolds because they can simulate the ECM structure of body tissues and provide biochemical signals to support cell proliferation and differentiation. Optical crosslinking has been widely considered for the fabrication of tissue engineering polymer scaffolds. This is due to their rapid curing under biological conditions at physiological temperatures with special control over gel kinetics, which enable these hydrogels to fabricate scaffolds with complex shapes. For hydrogels used in biomedical and tissue engineering, it is necessary that their mechanical properties be similar to natural tissue [37].

\section{Wound Dressing}

Min Hee Kim and his colleagues were able to make MC methylcellulose hydrogels containing silver oxide particles as wound dressings. They used Sprague four-week-old field hairs for this experiment. Field was performed under controlled conditions of temperature, humidity and light [38]. Their histopathological examina- tion showed that the part of the wound treated with MC hydrogel containing silver oxide nanoparticles was more successful than the untreated wound. It has been reported that the healing rate of burn wounds with MC hydrogel is significantly increased [38].The treatment pattern of burn wounds was also studied to examine untreated tissue. Particles were evaluated by biological dyes on days 1,3 , 7,14 , and 21 . The results showed that MC hydrogels without silver nanoparticles caused inflammation and tissue necrosis, while hydrogels containing silver nanoparticles without inflammation and necrosis [38]. Hydrogel-based dressings can absorb water several times their dry weight and for Absorbing wound secretions and helping to reduce the temperature at the wound site and creating a moist environment will be helpful. Existing wound dressings have disadvantages such as lack of antibacterial activity, insufficient oxygen permeability and water permeability and poor mechanical properties [39]. To overcome these problems, Hassan Namazi and his colleagues used a nanocomposite hydrogel in which antibiotics were used.

Has been designed through the combination of mesoporous silica and carboxymethylcellulose as a nanoparticle carrier. Tetracycline and methylene blue were injected into the system as antibacterial drugs and showed different release [39]. Tetracycline is a broad-spectrum antibiotic for the treatment of skin infections, acne, soft tissues and wounds. Tetracycline showed very effective topical treatment in bacterial skin infections [39]. Methylene blue is known as a cationic dye with antiseptic properties that is used in humans to treat poisoning, kidney stones, methemoglobin and cyanide. In addition, it is widely used as a light-sensitive substance in photodynamic therapy, which is an emerging way to fight antibiotic-resistant bacteria $[39,40]$.

\section{Drug Release}

Abdullahi et al. Succeeded in making a hydrogel loaded with fluoxamine by ultrasound, which showed the controlled release of the drug in the simulated environment of the body. They believe that the mechanism of drug release from the hydrogel depends on various factors such as the composition of the hydrogel, its geometric structure, method of preparation, type of drug and environmental conditions during the release period, $\mathrm{pH}$ is one of the most important factors [41]. In another experiment, Ganji, et al. Succeeded in slowly releasing pyridostigmine bromide using injectable hydrogel, which is sensitive to chitosan temperature in vitro. They believe that by adding glycerol phosphate salt, a darker solution was observed than the unsalted chitosan solution. Turbidity changes of chitosan and chitosan / glycerol phosphate solutions have been shown over time for solutions containing $8 \%$ by weight / volume of salt. In chitosan solution, the turbidity of the solution did not change significantly over time at $37^{\circ} \mathrm{C}$. Therefore, it can be concluded that chitosan solution without glycerol phosphate salt is not temperature sensitive and remains unchanged for a long time at 37 
${ }^{\circ}$ C. However, in the chitosan / glycerol phosphate solution, a slight increase in turbidity was observed over time at $37^{\circ} \mathrm{C}$. After 9 minutes, the darkness suddenly increased. The point at which a sudden increase in opacity is observed is the point of gel formation. This experiment showed that the addition of glycerol phosphate salt to the chitosan solution reduced the stability of this solution at $37^{\circ} \mathrm{C}$ and changed the phase from the solution state to the gel state in a short period of time [42].

Bakhshashi et al. Succeeded in constructing Baghdadit-vancomycin nanostructured scaffold with drug release capability, antibacterial activity and biocompatibility, and drug release was evaluated on a net basis. In their study, the addition of vancomycin was recorded as a drug model in the Baghdad scaffold for drug release behavior [43]. The mechanism of drug release from the scaffold was observed both explosively and in a controlled manner after immersion in phosphate-buffered saline solution (PBS). Specifically, during the first 6 hours of explosive release and after a period of time, a Stable release was observed, however, 45 to $75 \%$ of the drug was removed from the scaffold after 36 hours for all drug compounds. Vancomycin release killed S. aureus and was able to prevent them from replicating on the scaffold. They reported that $35 \%$ of the drug remained in the scaffold and was not removed, so it can be argued that bacterial infection in the early stages of bone infection can be controlled with Baghdadit / vancomycin scaffold [43-45].

\section{Conclusion}

In the present review article, the types of hydrogels and their properties, their formation mechanism and medical application were discussed. Regarding the mechanism of hydrogel formation, there are different methods for crosslinking the structure. Physically networked hydrogels have been used as a substrate for tissue engineering for a variety of medical applications, including the release of bioactive substances and the confinement of cells. The advantage of these networks is the lack of use of organic solvents. Release of pharmaceutical agents from hydrogels is mainly done by swelling of the structure. In recent years, attention to stimulus-responsive hydrogels has increased. Medications such as temperature and $\mathrm{pH}$ play a role in the controlled release of pharmaceutical agents from the hydrogel network. Hydrogels are considered as an attractive biomaterial for various medical applications due to their water absorption, soft structure, biocompatibility and similarity to ECM.

\section{Acknowledgment}

This scientific report was supported and financed by the Danesh Paper Company (DPC).

\section{References}

1. Nguyen QuangVinh, Dai Phu Huynh, Jae Hyung Park, Doo Sung Lee (2015) Injectable polymeric hydrogels for the delivery of therapeutic agents: A review. European Polymer J 72: 602-619.
2. Janani Radhakrishnan, Uma Maheswari Krishnan, Swaminathan Sethuraman (2014) Hydrogel based injectable scaffolds for cardiac tissue regeneration. Biotech Adv (32)2: 449-461.

3. Thambi Thavasyappan, Doo Sung Lee (2017) Injectable hydrogels for sustained release of therapeutic agents. J Controlled Release 267: 57-66.

4. Hoffman Allan S (2012) Hydrogels for biomedical applications. Adv Drug Del Rev 64:18-23.

5. FaheemAkhtar Muhammad, Muhammad Hanif, Nazar Muhammad Ranjha (2016) Methods of synthesis of hydrogels A review. Saudi Pharm J 24, 554-559.

6. Ansuja Pulickal Mathew, Saji Uthaman, Ki Hyun Cho, Chong Su Cho, In Kyu Park (2018) Injectable hydrogels for delivering biotherapeutic molecules. Inter J Bio Macromolecules 110: 17-29.

7. Michael S Riederer, Brennan D Requist, Karin A Payne, J Douglas Way, Melissa D Krebs (2016) Injectable and microporous scaffold of densely packed, growth factor encapsulating chitosan microgels. Carbohydrate Polymers 152: 792-801.

8. Settimio Pacelli, Francisca Acosta, Aparna R Chakravarti , Saheli G Samanta , Jonathan Whitlow, et al. (2017) Nanodiamond based injectable hydrogel for sustained growth factor release: Preparation, characterization and in vitro analysis. Acta Biomaterialia 58: 479-491.

9. Jeanie L Drury, David J (2003) Mooney Hydrogels for tissue engineering: scaffold design variables and applications. Biomaterials, 24: 43374351.

10. Ying Luo, James B Kobler, James T Heaton, Xinqiao Jia, Steven M Zeitels (2010) Injectable Hyaluronic Acid Dextran Hydrogels and Effects of Implantation in Ferret Vocal Fold. Wiley Inter Science 93(2): 386-393.

11. Masoud Ghorbani, Jafar Ai, Mohammad Reza Nourani , Mahmoud Azami , Batool Hashemi Beni (2017) Injectable natural polymer compound for tissue engineering of intervertebral disc: In vitro study. Mater Sci Engineering 80: 502-508.

12. Amit Alexander , Ajazuddin , Junaid Khan, Swarnlata Saraf, Shailendra Saraf (2014) 5 Polyethylene glycol (PEG)-Poly(N isopropylacrylamide) (PNIPAAm) based thermosensitive injectable hydrogels for biomedical applications. Eur J Pharm Biopharm 88: 575-585.

13. James D Kretlow, Leda Klouda, Antonios G Mikos (2007) Injectable matrices and scaffolds for drug delivery in tissue engineering. Adv Drug Deliv Rev 59, 263-273.

14. Kai Wang , Zongchao Han (2017) Injectable hydrogels for ophthalmic applications. J Controlled Release 268: 212-224.

15. Jundika C. Kurnia, Erik Birgersson, Arun S. Mujumdar (2012) Analysis of a model for $\mathrm{pH}$ sensitive hydrogels. Polymer 53(2): 613-622.

16. Muhammad Faheem Akhtar, Muhammad Hanif, Nazar Muhammad Ranjha (2016) Methods of synthesis of hydrogels... A review. Saudi Pharm J 24(5): 554-559.

17.Xuping Qin, Fang Zhao, Yingkai Liu, Shengyu Feng (2011) Frontal photopolymerization synthesis of multilayer hydrogels with high mechanical strength. Europ Polym J 47(10): 1903-1911.

18. Nelson Monteiro, Greeshma Thrivikraman , Avathamsa Athirasala, Anthony Tahayeri, Cristiane M França, et al. (2017) Photopolymerization of cell laden gelatinmethacryloyl hydrogels using a dental curing lightfor regenerative dentistry. Dental materials 34(3): 389-399.

19. Iris Mironi Harpaz, Dennis Yingquan Wang, Subbu Venkatraman, Dror Seliktar (2012) Photopolymerization of cell encapsulating hydrogels: Crosslinking efficiency versus cytotoxicity. Acta Biomaterialia 8(5): 1838-1848.

20. 0. Guaresti , C. Garcia-Astrain, R.H. Aguirresarobe , A. Eceiza , N. Gabilondo (2018). Synthesis of stimuli-responsive chitosan-based hydrogels by Diels-Alder cross-linking 'click' reaction as potential carriers for drug administration. Carbohydrate Polymers 183: 278-286. 
21. Yangling Li (2016) A biodegradable starch hydrogel synthesized via thiolene click chemistry, Polymer Degradation and Stability, PDST 8017.

22. Ruobing Bai, Quansan Yang, Jingda Tang, Xavier P. Morelle, Joost Vlassak, et al. (2017) Fatigue fracture of tough hydrogels. Extreme Mechanics Letters 15: 91-96.

23. Lingyu Wei, Jinjin Chen, Shuhua Zhao, Jianxun Ding, Xuesi Chen (2017) Thermo sensitive polypeptide hydrogel for locally sequential delivery of two pronged antitumor drugs. Acta Biomater 58: 44-53.

24. Xuebin Ma , Tingting Xu, Wei Chen, Hongye Qin, Bo Chi, et al. (2018) Injectable hydrogels based on the hyaluronic acid and poly $(\gamma$ glutamic acid) for controlled protein delivery. Carbohydr Polym 179: 100-109.

25. Liefeng Hu, Panpan Zhang, Xin Wang , Xu Cheng , Jiejie Qin, et al. (2017) $\mathrm{pH}$ sensitive carboxymethyl chitosan hydrogels via acid labile ortho ester linkage for potential biomedical applications. Carbohydr Polym, 178: 166-179.

26. Bin Jeremiah D. Barba, Charito Tranquilan Aranilla, Lucille V. Abad (2016) Hemostatic potential of natural/synthetic polymer based hydrogels crosslinked by gamma radiation. Radiation Physics and Chemistry 118: 111-113.

27. Desiree Alesa Gyles, Lorena Diniz Castro, Jose Otavio Carrera Silva Roseane Maria Ribeiro Costa (2017) A review of the designs and prominent biomedical advances of natural and synthetic hydrogel formulations. European Polym J 88: 373-392.

28. Mokhamad Nur, Todor Vasiljevic (2017) Can natural polymers assist in delivering insulin orally ?. International J Biological Macromolecules 103: 889-901.

29. Iwona Cicha, Rainer Detsch, Raminder Singh, Supachai Reakasame, Christoph Alexiou, et al. (2017) Biofabrication of vessel grafts based on natural Hydrogels. Biomedical Engineering 2: 83-89.

30. Weissleder R \& Bogdanov A (1996). U.S. Patent No. 5,514,379. Washington, DC: U.S. Patent and Trademark Office.

31. Armin Vedadghavami, Farnaz Minooei, Mohammad Hossein Mohammadi , Sultan Khetani, Ahmad Rezaei Kolahchi, et al. (2017) Manufacturing of hydrogel biomaterials with controlled mechanical properties for tissue engineering applications. Acta Biomate, 15(62) :42-63.

32. Muhammad Abdul Haq, Yunlan Su, Dujin Wang (2017) Mechanical properties of PNIPAM based hydrogels: A review. Mater Sci Eng C 1(70): 842-855.

33. Reza Abdollahi, Mohammad Taghi Taghizadeh , Samira Savani (2018) Thermal and mechanical properties of grapheme oxide nanocomposite hydrogel based on poly (acrylic acid) grafted onto amylose. Polym Degradation and Stability 147: 151-158.
34. Haipeng Li, Xiaoqing Song, Baoe Li, Jianli Kang , Chunyong Liang , Hongshui Wang, et al. (2017) Carbon nanotube reinforced mesoporous hydroxyapatite composites with excellent mechanical and biological properties for bone replacement material application. Materials Science and Engineering C 1(77): 1078-1087.

35. Sheva Naahidi, Mousa Jafari, Megan Logan, Yujie Wang, Yongfang Yuan, et al. (2017) Biocompatibility of hydrogel based scaffolds for tissue engineering. Applications. Biotechnol Adv JBA 7127 35(5): 530-544.

36. Jingzhou Yang, Yu Shrike Zhang, Kan Yue, Ali Khademhosseini (2017) Cell Laden Hydrogels for Osteochondral and Cartilage Tissue Engineering. Acta Biomater, ACTBIO 4671 15(57): 1-25.

37. Yingshan Zhou, Shuyan Zhao, Can Zhang, Kaili Liang, Jun Li, et al. (2018) Photopolymerized Maleilated Chitosan/Thiolterminated Poly (vinyl alcohol) hydrogels as Potential Tissue Engineering Scaffolds. carbohydr polym CARP 13159 15(184): 383-389.

38. Min Hee Kim, Hanna Park, Hyung Chan Nam, Se Ra Park, Ju Young Jung, et al. (2018) Injectable methylcellulose hydrogel containing silver oxide nanoparticles for burn wound healing. Carbohydrate Polymers 181: 579-586.

39. Hassan Namazi, Rasul Rakhshaei, Hamed Hamishehkar, Hossein Samadi Kafil (2015) Antibiotic loaded carboxymethylcellulose/ MCM 41 nanocomposite hydrogel films as potential wound dressing. International J Biological Macromolecules, 85: 327-334.

40. Elbadawy A Kamoun, El Refaie S Kenawy, Xin Chen (2017) A review on polymeric hydrogel membranes for wound dressing applications: PVA based hydrogel dressings. J Adv R 8(3): 217-233

41. Enas M Ahmed (2015) Hydrogel: Preparation, characterization, and applications: A review. J adv res 6(2): 105-121.

42. Oyen M L (2014) Mechanical characterisation of hydrogel materials. International Materials Reviews 59(1): 44-59.

43. H.R. Bakhsheshi Rad, E. Hamzah , A.F. Ismail , M. Aziz , Z. Hadisi, et al. (2017) Novel nanostructured baghdadite vancomycin scaffolds: In vitro drug release, antibacterial activity and biocompatibility. Materials Letters 209: 369-372.

44. Li Xinming, Cui Yingde, Andrew W Lloyd, Sergey V Mikhalovsky, Susan R Sandeman, et al. (2008) Polymeric hydrogels for novel contact lens based ophthalmic drug delivery systems: A review. Contact Lens \& Anterior Eye 31(2): 57-64.

45. Susanne Kirchhof, Achim M Goepferich, Ferdinand P Brandl (2015) Hydrogels in ophthalmic applications. Eur J Pharms Biopharm, 95: 227 238. 